\title{
Oscillatory Failure Case Detection For Aircraft Using an Adaptive Sliding Mode Differentiator Scheme
}

\author{
Halim Alwi and Christopher Edwards
}

\begin{abstract}
This paper proposes a nonlinear scheme for estimating an Oscillatory Failure Case (OFC) using a nonlinear model of the hydraulic actuator which requires an estimate of rod speed. The estimation of the rod speed is provided by a supertwist differentiator. The supertwist gains are allowed to adapt to maintain sliding during high frequency and high amplitude OFCs. Ideally low gains in the differentiator are required because of the noisy environment associated with the physical system. A Lyapunov based analysis for the novel adaptive supertwist scheme is also presented. Simulations on both liquid and solid OFCs have been carried out on the full nonlinear aircraft model with highly detailed models of the actuators which include sensor and process noise. Good estimation of both actuator rod speed and the OFC is obtained.
\end{abstract}

\section{INTRODUCTION}

The study of Oscillatory Failure Case (OFC) problems has become much more important with the introduction of Electronic Flight Control Systems (EFCS). Early work on OFC which appears in [3] is motivated by the study of the effect of EFCS failures on the structural load on an aircraft. OFC is a type of EFCS failure which can cause a significant increase in the structural load due to erroneous oscillation. When coupled with the flexible modes of the structure, OFC can generate resonance phenomenon and cause unacceptably high vibration and loads [6]. Faulty electronic components is the main source of OFC. These generate erroneous sinusoidal signals which propagate through the actuator control loop between the Flight Control Computer (FCC) and the control surface [6]. There are two types of OFC: termed liquid and solid [3], [6]. The liquid OFC behaves as an additive fault, whereby the OFC signal adds to the desired commanded position from the FCC and the control surface tracks the corrupted demand signal. For the solid OFC case, the surface is 'disconnected' from the FCC and the demand signal is replaced totally by the OFC signal and the control surface executes a pure uncommanded periodic motion (and does not respond to the demanded rod position) [3], [6].

An interesting detection scheme from an industrial perspective is given in [6]. This paper discusses a scheme for OFC detection which has been validated and implemented (with low computational load) on the AIRBUS A380 which is currently in service. The design is model-based and the rod position is approximated from an analytical model and is compared with the actual measurement to create a residual signal. Both liquid and solid OFC faults can be detected by the scheme.

H. Alwi and C. Edwards are with the Control Research Group, Department of Engineering, University of Leicester, University Road, Leicester, LE1 7RH, UK ha18, ce14@le.ac.uk
In [1], [2], a nonlinear design method is proposed. The method uses a nonlinear analytical model of the actuator system together with an observer to obtain a residual. Detection is obtained by evaluating the residual signal in a similar way to the methodology in [6]. Other recent results are given in [13], [12], [1], [7], [8], [11].

The scheme proposed in this paper presents a new nonlinear based method for estimating OFC. The idea is to manipulate the (analytical) mathematical model of the actuator to obtain an expression of the OFC signal. Most of the parameters used in the manipulated nonlinear equation are available through direct measurement, except for the actuator rod speed. In this paper, a novel adaptive sliding mode Super Twist Algorithm (STA), which is based on a Lyapunov approach will be used to estimate the rod speed. In terms of the STA, the work in [10], [4] represents the state of the art in terms of the use of a Lyapunov based approach for STA. The STA that will be used in this paper, is different to the one in [10], [4]. Here it is proposed that the gains for the STA are allowed to adapt based on the 'quality' of the sliding mode which is obtained. A Lyapunov analysis for the STA with adaptive gains proposed in this paper is provided. The adaptation scheme is motivated by the requirement for good reconstruction of the OFC. Because of the noisy situation in which the actuator operates, it is essential the gains of the STA are as low as possible. This motivates the need for the adaptation scheme. During fault free conditions and for low frequency and amplitude OFC, only a low STA gain is required to obtain a good reconstruction of the OFC. However for high amplitude and frequency OFC, a high gain is required.

The results presented here are part of ongoing work in an EU funded project called ADDSAFE, which aims to study advanced FDI for aircraft. The OFC scenario is one of the considered failure modes in this study. Simulation results using the ADDSAFE benchmark model [5] provided by AIRBUS are presented.

\section{Preliminary}

\section{A. Modelling of hydraulic actuator}

The hydraulic actuator model from [5], [6] is given by

$$
\dot{x}(t)=V_{c}(t)\left(\frac{\Delta_{p}(t)-\operatorname{sign}(i(t)) \frac{F_{\text {aero }}(t)}{S}}{\Delta_{p_{\text {ref }}}+\frac{K_{d}(t)}{S} V_{c}^{2}(t)}\right)^{\frac{1}{2}}
$$

where nominally

$$
V_{c}(t)=K_{c} i(t)
$$


where $K_{c}$ is a conversion factor from electrical current ( $\left.m A\right)$ to speed $(\mathrm{mm} / \mathrm{s})$. The current $i(t)$ is given by

$$
i(t)=K(u(t)-x(t))
$$

where $K$ is the (fixed) servo control gain. The signal $x(t)$ is the hydraulic actuator rod position and $u(t)$ is the commanded (from the FCC) rod position. The fixed constants are $\Delta_{p_{\text {ref }}}$ which is the differential pressure corresponding to the maximum rod speed, and $S$ which is the piston surface area. The parameters which depend on varying operational conditions (e.g. fluid temperature and/or the number of actuators used simultaneously on a given hydraulic circuit) are $\Delta_{p}(t)$ which is the actual hydraulic pressure delivered to the actuator, $F_{\text {aero }}(t)$ which is the aerodynamic forces applied on the control surface and $K_{d}(t)$ which is the adjacent actuator damping coefficient (in the case of 2 actuators per control surface).

\section{B. OFC modelling}

An OFC is caused by faults in any digital component (which generate unwanted sinusoidal signals) in the actuator control loop between the FCC and the control surface. These oscillations consequently propagate within the loop [6]. As in [6] only an OFC located in the servo control loop is considered. Specifically, it is assumed that the OFC source is in the analogic output signal between the FCC and the actuator (See Figure 1). In the ADDSAFE model, the OFC affects the computed/desired rod speed $V_{c}(t)$ so that

$$
V_{c}(t)= \begin{cases}V_{0}(t) & \text { nominal } \\ V_{0}(t)+K_{c} f_{\text {liq }}(t) & \text { liquid OFC } \\ K_{c} f_{\text {sol }}(t) \text { i.e. } V_{0}(t)=0 & \text { solid OFC }\end{cases}
$$

where

$$
V_{0}(t)=K_{c} K(u(t)-x(t))
$$

As in [6], the OFC signals are considered as sinusoids with amplitude and frequency uniformly distributed over the range of $1-10 \mathrm{~Hz}$.Beyond $10 \mathrm{~Hz}$, the OFC has no effect on control surface oscillation due to the low pass characteristics of the actuator. As shown in (4) the liquid OFC behaves as an additive fault, and the OFC signal adds to the desired position from the FCC and hence the control surface tracks the corrupted demand signal. As shown in (4), a more severe case is the solid OFC, where the demand surface position is replaced totally by the OFC signal. In this case, the control surface is 'disconnected' totally from the FCC and does not respond to the desired rod position, but instead behaves as a pure periodic motion. This is more severe, as any attempt to damp the oscillation does not have any impact as the control surface is 'disconnected' from any demand signal from the FCC [3], [6].

\section{OFC ESTIMATION}

In this section, the OFC estimation scheme will be presented. The idea is to rearrange the nonlinear equations (1), (3) and (4) such that the OFC signal can be estimated directly.

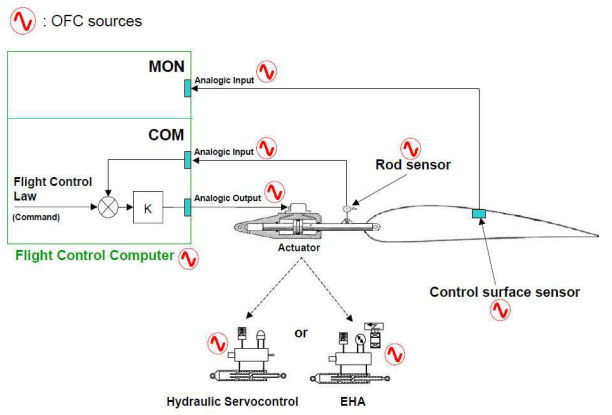

Fig. 1. Sources of OFC in the servo control loop [6]

Consider initially the liquid OFC case. By algebraic rearrangement (1) and (4) are equivalent to

$f_{\text {liq }}(t)=\frac{\dot{x}(t)\left(\frac{\Delta_{p_{\text {ref }}}}{\Delta_{p}(t)-\operatorname{sign}(i(t)) \frac{F_{\text {aero }}(t)}{S}-\dot{x}^{2}(t) \frac{K_{d}(t)}{S}}\right)^{\frac{1}{2}}-V_{0}(t)}{K_{c}}$

All the variables on the right hand side of (6) are available (measured) except for the actuator rod speed $\dot{x}(t)$.

For the solid OFC case, since $V_{0}=0$ in (4), similar arguments give the estimate of the solid OFC as

$$
f_{\text {sol }}(t)=\frac{\dot{x}(t)\left(\frac{\Delta_{p_{\text {ref }}}}{\Delta_{p}(t)-\operatorname{sign}(i(t)) \frac{F_{\text {aero }}(t)}{S}-\dot{x}^{2}(t) \frac{K_{d}(t)}{S}}\right)^{\frac{1}{2}}}{K_{c}}
$$

From (6) and (7), all the parameters are available (i.e., measured or assumed to be fixed) except for the actuator rod speed $\dot{x}(t)$. The idea in this paper is to use the supertwist differentiator (similar to [9]) to provide a robust estimate of rod speed from the measurement of rod position $x(t)$. Once $\dot{x}(t)$ is estimated, the OFC can be estimated using (6) and (7) since all the values on the right hand side are known.

\section{A. Estimation of actuator rod speed}

In this section, a formulation of the Levant differentiator [9] based on the STA is considered. A novel aspect of the formulation is that the gains will be allowed to adapt. This will ensure a good estimate of rod speed even in the presence of an OFC, which therefore allows in turn good reconstruction of the OFC from (6) and (7). In fault free conditions, a 'small gain' is sufficient to ensure a good estimate of rod speed. The 'small' gains ensure the effect of noise is not accentuated. For this particular application, this is very important since the observer must function in a noisy environment. However, when an OFC occurs, although the detection of the OFC is still possible, sliding is broken thus compromising the estimate of the rod speed. In this case, larger gains are required to ensure a sliding motion is maintained to provide a good rod speed estimate.

Consider equation (1) as a special case of the equation

$$
\dot{x}(t)=f(t, x)
$$

with measured output $y(t)=x(t)$. Assume that the time derivative of the function on the right hand side of (8) is bounded i.e.,

$$
|\dot{f}(t, x)| \leq \delta
$$


for some unknown constant $\delta>0$.

Consider an observer with the following structure

$$
\begin{aligned}
& \dot{z}_{1}(t)=-\alpha(t)\left|e_{1}(t)\right|^{1 / 2} \operatorname{sign}\left(e_{1}(t)\right)+z_{2}(t) \\
& \dot{z}_{2}(t)=-\beta(t) \operatorname{sign}\left(e_{1}(t)\right)
\end{aligned}
$$

where $e_{1}(t)=z_{1}(t)-x(t)$. Subtracting (8) from (10) yields the error system

$$
\begin{aligned}
& \dot{e}_{1}(t)=-\alpha(t)\left|e_{1}(t)\right|^{1 / 2} \operatorname{sign}\left(e_{1}(t)\right)+z_{2}(t)-f(t, x) \\
& \dot{z}_{2}(t)=-\beta(t) \operatorname{sign}\left(e_{1}(t)\right)
\end{aligned}
$$

Consider $e_{2}(t)=z_{2}(t)-f(t, x)$, then (12)-(13) can be written as

$$
\begin{aligned}
& \dot{e}_{1}(t)=-\alpha(t)\left|e_{1}(t)\right|^{1 / 2} \operatorname{sign}\left(e_{1}(t)\right)+e_{2}(t) \\
& \dot{e}_{2}(t)=-\beta(t) \operatorname{sign}\left(e_{1}(t)\right)-\dot{f}(t, x)
\end{aligned}
$$

If a 2nd order sliding motion is induced $e_{1}(t)=\dot{e}_{1}(t)=0$ and therefore from (14), $e_{2}(t)=0 \Rightarrow z_{2}(t)=f(t, x)$ and therefore $z_{2}(t)$ from (11) provides the estimate of rod speed since $f(t, x)=\dot{x}(t)$.

In this paper, the gains $\alpha(t)$ and $\beta(t)$ are chosen as:

$$
\begin{aligned}
& \alpha(t)=\sqrt{2 L(t)} \\
& \beta(t)=4 L(t)
\end{aligned}
$$

for some time varying scalar $L(t)$ which will be described in the sequel. Define

$$
L(t)=r(t)+\ell
$$

where the variable $\ell$ is a fixed positive scalar while the varying $r(t)$ (also positive) is adapted according to

$$
\dot{r}(t)= \begin{cases}\gamma\left|e_{1}(t)\right|^{1 / 2} & \text { if } r(t) \leq r_{\max } \\ 0 & \text { otherwise }\end{cases}
$$

where $\gamma>0$ is a positive design constant and the scalar $r_{\max } \gg \delta$. The control law in (19) allows $r(t)$ to adapt until the value $r(t)=r_{\max }$ is reached.

Proposition 1: The adaptive gains from (16)-(19) ensure the error system (14)-(15) converges $\left\{e_{1}(t)=0, e_{2}(t)=0\right\}$.

Proof: See Appendix.

\section{B. Practical Implementation}

For practical implementation, ideal sliding is not achievable due to noise and therefore the adaptation rule in (19) is not practical. In this paper, it has been modified to

$$
\dot{r}(t)= \begin{cases}\gamma D\left(\left|e_{1}(t)\right|^{1 / 2}\right) & \text { if } r(t) \leq r_{\max } \\ 0 & \text { otherwise }\end{cases}
$$

where the function $D(z): \mathbb{R} \mapsto \mathbb{R}$ is the dead-zone

$$
D(z)= \begin{cases}0 & \text { if }|z|<\varepsilon \\ z & \text { otherwise }\end{cases}
$$

where $\varepsilon$ is a positive scalar. The idea here is to adapt the gains when $\left|e_{1}(t)\right|^{1 / 2}$ unacceptably deviates from zero. The gain $r(t)$ will increase in magnitude according to (20) to force $e_{1}(t)$ back into a sliding regime.

Proposition 2: Using the adaptation rule (20) ensures the error system (14)-(15) and $r(t)$ remains bounded.

Proof: See Appendix.

\section{Simulations}

The scheme proposed above has been tested on an ADDSAFE benchmark model provided by AIRBUS [5]. The nonlinear high fidelity model represents a generic twin engine civil transport aircraft and incorporates highly detailed nonlinear actuator models. The simulations are conducted at an altitude of $30000 \mathrm{ft}$, a speed of Mach 0.64 (241kts), a weight of 200 tonnes and centre of gravity of 30\% MAC. In the benchmark model, the actuators are represented as high fidelity nonlinear models and the parameters $\Delta_{p}, F_{\text {aero }}$ and $K_{d}$ vary based on changes in the operational conditions. For the observer design, these parameters are assumed to be fixed at their mean values.

As mentioned earlier, the OFC is considered to appear in the analogic output of the FCC used to send the electrical current signal which provides the desired actuator position to the solenoid valve of the actuator. Here, the control surface considered is the left elevator.

The sliding mode supertwist differentiator design parameters from (18) and (20) used in the simulation are $\gamma=3 \times 10^{4}$, $\ell=12$ and $\varepsilon=0.45$. The simulation was performed using a solver with a fixed time step of $0.01 \mathrm{~s}$. For added realism, sensor and process noise have been considered in the simulation.

\section{A. Simulation Results}

Various OFC amplitudes and frequencies have been tested. For consistency and for comparison, all the tests were conducted using the same manoeuvre whereby the aircraft is given a pilot longitudinal stick input as shown in Figure 2 (a). For all tests, the OFC occurs at $10 \mathrm{sec}$. For brevity, the results shown here represent the extreme cases of low and high amplitude and frequency to highlight the performance of the proposed scheme.

1) Fault Free: Figure 2 shows the fault free scenario. Figure 2(a) shows the doublet input applied to the pilot longitudinal stick to excite the system. The left elevator deflection is also given in this figure. Figure 2(b) shows the performance of the sliding mode supertwist differentiator. The plot of $L(t)$ shows that no adaptation takes place in the nominal case. Figure 2(c) shows a good estimate of rod speed $\dot{x}(t)$ (solid blue line) compared to the actual rod speed (red dashed line). Figure 2(c) shows no OFC is present.

2) Solid OFC: Figure 3 shows a solid OFC of amplitude $1 \mathrm{deg}$ and frequency of $7 \mathrm{~Hz}$. Due to the high frequency oscillation, zoomed-in plots $(9-12 \mathrm{sec})$ are shown to illustrate the effectiveness of the scheme. Figure 3(a) shows the effect of a solid OFC on the left elevator. Again the OFC totally replaces the commanded signal from the FCC (red dashed line). Figure 3(b) shows the gain $L(t)$ adapting when sliding degrades once the OFC occurs. The adaptation ensures that sliding is reestablished. Figure 3(c) shows the rod speed and OFC estimate for the high frequency case in the presence of noise. When the OFC occurs, the quality of the estimate is compromised due to the high frequency of the OFC oscillation. Here, the higher supertwist gain $L(t)$ (from the adaptation) ensures good estimates (blue solid lines) of both rod speed and the OFC. The small spikes on the estimates are due to noise and the high frequency of the OFC. 

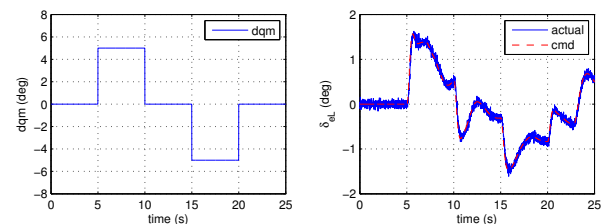

(a) pilot stick input and control surface deflection
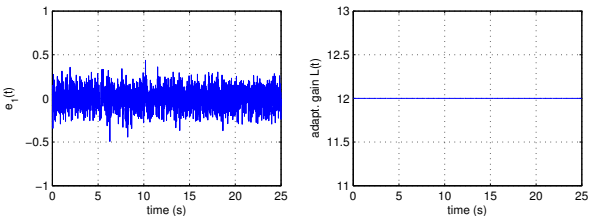

(b) supertwist performance
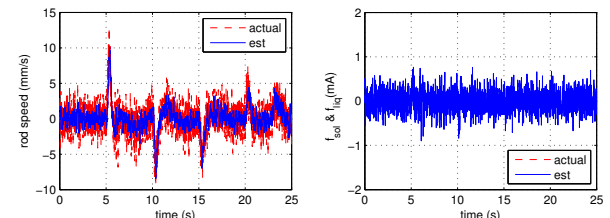

(c) estimations

Fig. 2. Fault free condition

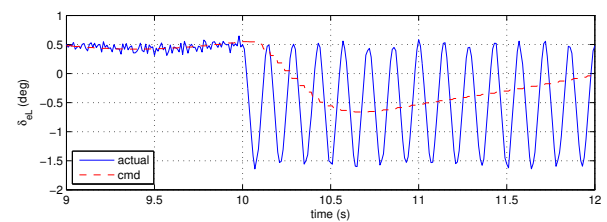

(a) control surface
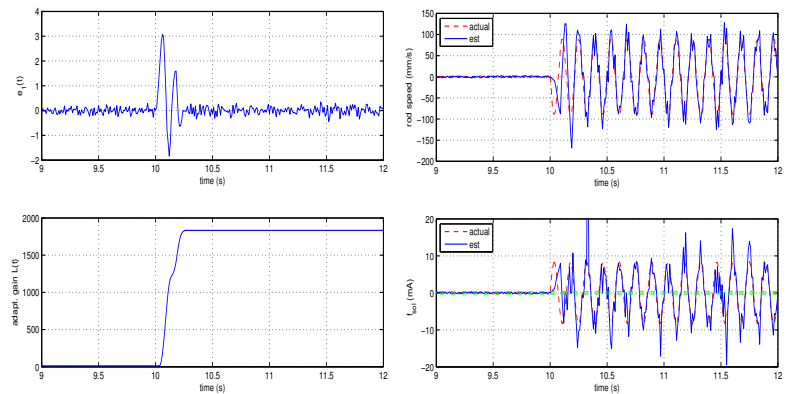

(b) supertwist performance

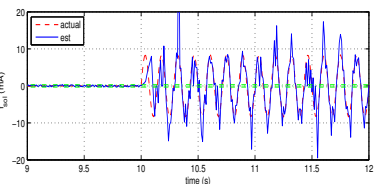

(c) estimations

Fig. 3. Solid OFC (amplitude 1.0, frequency 7.0)

Note that for the solid OFC case, the rod speed estimate shown in Figure 3(c) can also be used to confirm the occurrence of an OFC. This is due to the fact that for solid OFC, the fault signal totally replaces the commanded signal, and the left elevator (and the actuator rod speed) performs a purely oscillatory motion.

3) Liquid OFC: Figure 4 shows the case of an amplitude of $0.5 \mathrm{deg}$ at a frequency of $0.5 \mathrm{~Hz}$. Figure $4(\mathrm{a})$ shows the effect of a liquid OFC on the left elevator deflection (blue solid line). Here the OFC signal adds to the demanded signal (red dashed line) from the FCC. Figure 4(b) shows that for this combination of low amplitude and frequency, no degradation in the sliding motion occurs and therefore adaptation to the supertwist gain $L(t)$ is not required. A good estimate of

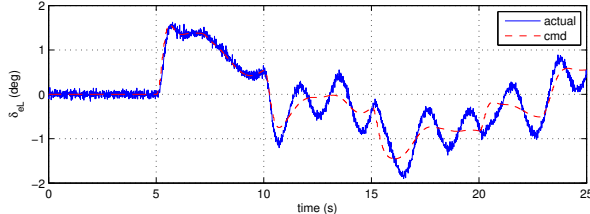

(a) control surface
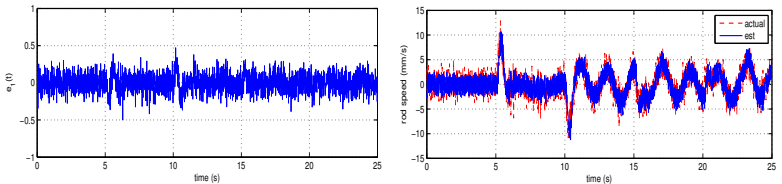

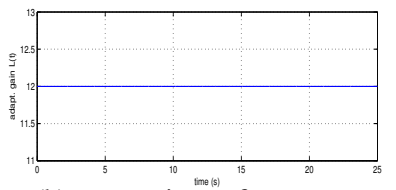

(b) supertwist performance

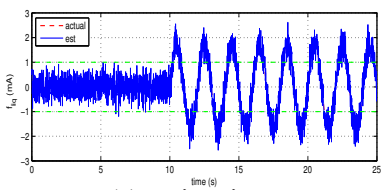

(c) estimations
Fig. 4. Liquid OFC (amplitude 0.5, frequency 0.5)

rod speed $\dot{x}$ is shown in Figure 4(c) (solid blue line) which overlaps the actual rod speed (red dashed line). Figure 4(c) shows a good estimate of the OFC (blue solid line) compared with the actual OFC (red dashed line).

\section{Conclusions}

This paper has presented an OFC detection scheme. The scheme uses an estimate of the actuator rod speed from an adaptive supertwist observer scheme. An analysis for the novel adaptive supertwist scheme used in this paper uses a Lyapunov based approach. The supertwist gains are allowed to adapt to maintain sliding during high frequency and high amplitude OFC conditions. Simulation results based on the full nonlinear model of the aircraft using a highly detailed model of the actuators have been carried out. Both liquid and solid OFC cases have been considered. The results show good estimates of both the actuator rod speed and the OFC.

\section{ACKNOWLEDGEMENT}

The authors are grateful for the provision of an EU FP7 grant ADDSAFE (FP7-233815) which funded this work.

\section{APPENDIX}

Proof of Proposition 1: Consider the following Lyapunov function candidate for the error system (14) and (15)

$$
\begin{aligned}
V(t, \zeta)= & \frac{1}{L^{3 / 2}(t)} \zeta(t)^{\mathrm{T}} P(t) \zeta(t) \\
& +\frac{\sqrt{2}}{2 \gamma} e_{r}(t)\left(4 L^{-1}(t)+\delta L^{-2}(t)\right) e_{r}(t)
\end{aligned}
$$

where

$$
e_{r}(t)=r(t)-\delta
$$

and

$$
\zeta^{\mathrm{T}}(t):=\left[\left|e_{1}(t)\right|^{1 / 2} \operatorname{sgn}\left(e_{1}(t)\right) \quad e_{2}(t)\right]
$$


The time varying matrix

$$
P(t)=\frac{1}{2}\left[\begin{array}{cc}
4 \beta(t)+\alpha^{2}(t) & -\alpha(t) \\
-\alpha(t) & 2
\end{array}\right]
$$

is positive definite since $\beta(t)>0$ and $\operatorname{det}(P(t))>0$. Taking the derivative of (22) yields

$$
\begin{aligned}
\dot{V}(t)= & \underbrace{\frac{d}{d t}\left(\frac{1}{L^{3 / 2}(t)} \zeta(t)^{\mathrm{T}} P(t) \zeta(t)\right)}_{\dot{V}_{1}} \\
& +\underbrace{\frac{d}{d t}\left(\frac{\sqrt{2}}{2 \gamma} e_{r}(t)\left(4 L^{-1}(t)+\delta L^{-2}(t)\right) e_{r}(t)\right)}_{\dot{V}_{2}}
\end{aligned}
$$

The first component can be expressed as

$$
\begin{aligned}
\dot{V}_{1}(t)= & \underbrace{\zeta^{\mathrm{T}}(t)\left(\frac{d}{d t}\left(\frac{1}{L^{3 / 2}(t)} P(t)\right)\right) \zeta(t)}_{\dot{V}_{1 a}} \\
& +\underbrace{\frac{1}{L^{3 / 2}(t)}\left(\dot{\zeta}(t)^{\mathrm{T}} P(t) \zeta(t)+\zeta(t)^{\mathrm{T}} P(t) \dot{\zeta}(t)\right)}_{\dot{V}_{1 b}}
\end{aligned}
$$

Consider initially the term $\dot{V}_{1 a}(t)$ in (27). Substituting for $\alpha(t)$ and $\beta(t)$ from (16)-(17) into $\dot{V}_{1 a}(t)$ gives

$$
\begin{aligned}
\dot{V}_{1 a}(t) & =\zeta^{\mathrm{T}}(t)\left(\frac{d}{d t} \frac{1}{2}\left[\begin{array}{cc}
18 L^{-1 / 2}(t) & -\sqrt{2} L^{-1}(t) \\
-\sqrt{2} L^{-1}(t) & 2 L^{-3 / 2}(t)
\end{array}\right]\right) \zeta(t) \\
& =\frac{1}{2} \zeta^{\mathrm{T}}(t) \underbrace{\left[\begin{array}{cc}
-9 L^{-3 / 2}(t) & \sqrt{2} L^{-2}(t) \\
\sqrt{2} L^{-2}(t) & -3 L^{-5 / 2}(t)
\end{array}\right]}_{\psi(t)} \dot{L}(t) \zeta(t)
\end{aligned}
$$

Notice that $\dot{L}(t) \geq 0$ since $\dot{L}(t)=\dot{r}(t)$ and from (19) $\dot{r}(t) \geq$ 0 for all time. Since $L(t)>0$ it can be easily shown that $\psi(t)<0$ and consequently since $\dot{L}(t) \geq 0$, it follows

$$
\dot{V}_{1 a}(t)=\frac{1}{2} \zeta^{\mathrm{T}}(t) \psi(t) \dot{L}(t) \zeta(t) \leq 0
$$

Arguing as in [10], the term $\dot{V}_{1 b}(t)$ in (27) is given by

$$
\begin{aligned}
\dot{V}_{1 b}(t)= & \frac{1}{L^{3 / 2}}\left(-\frac{1}{\left|e_{1}\right|^{1 / 2}} \zeta^{\mathrm{T}} Q \zeta\right. \\
& \left.-\dot{f}(t)\left(-\alpha(t)\left|e_{1}\right|^{1 / 2} \operatorname{sgn}\left(e_{1}\right)+2 e_{2}\right)\right)
\end{aligned}
$$

where

$$
Q(t)=\frac{\alpha(t)}{2}\left[\begin{array}{cc}
2 \beta(t)+\alpha^{2}(t) & -\alpha(t) \\
-\alpha(t) & 2
\end{array}\right]
$$

From (9), it is assumed that $\dot{f}(t, x)$ is bounded, and therefore using similar arguments to those in [10],

$$
\dot{V}_{1 b}(t) \leq \frac{1}{L^{3 / 2}}\left(-\frac{1}{\left|e_{1}\right|^{1 / 2}} \zeta^{\mathrm{T}} \tilde{Q} \zeta\right)
$$

where

$$
\tilde{Q}(t)=\frac{\alpha(t)}{2}\left[\begin{array}{cc}
2 \beta(t)+\alpha^{2}(t)-2 \delta & -\left(\alpha(t)+\frac{2 \delta}{\alpha(t)}\right) \\
-\left(\alpha(t)+\frac{2 \delta}{\alpha(t)}\right) & 1
\end{array}\right]
$$

substituting for $\zeta(t)$ equation (32) can be written as

$$
\begin{aligned}
\dot{V}_{1 b}(t) \leq & -\frac{\alpha}{2 L^{3 / 2}\left|e_{1}\right|^{1 / 2}}\left((10 L-2 \delta)\left|e_{1}\right|\right. \\
& \left.-\frac{4}{\sqrt{2 L}}(L+\delta) e_{2}\left|e_{1}\right|^{1 / 2} \operatorname{sgn}\left(e_{1}\right)+e_{2}^{2}\right)
\end{aligned}
$$

Using the fact that

$$
\begin{array}{r}
-\frac{4}{\sqrt{2 L}}(L+\delta) e_{2}\left|e_{1}\right|^{1 / 2} \operatorname{sgn}\left(e_{1}\right)+e_{2}^{2} \\
\equiv \tilde{e}^{2}-\left(2 L+4 \delta+2 \delta^{2} L^{-1}\right)\left|e_{1}\right|
\end{array}
$$

where

$$
\tilde{e}:=\left(-\frac{2}{\sqrt{2 L}}(L+\delta)\left|e_{1}\right|^{1 / 2} \operatorname{sgn}\left(e_{1}\right)+e_{2}\right)
$$

Substituting (35) into (34) yields

$$
\begin{aligned}
\dot{V}_{1 b}(t) & \leq-\frac{\alpha}{2 L^{3 / 2}\left|e_{1}\right|^{1 / 2}}\left(\tilde{e}^{2}+\left(8 L-6 \delta-2 \delta^{2} L^{-1}\right)\left|e_{1}\right|\right) \\
& =-\frac{\alpha}{2 L^{3 / 2}\left|e_{1}\right|^{1 / 2}}\left(\tilde{e}^{2}+\frac{2}{L}(4 L+\delta)(L-\delta)\left|e_{1}\right|\right)
\end{aligned}
$$

Using (16), (23) and the fact that $L-\delta=(r+\ell-\delta)=\ell+e_{r}$, (37) can be written as

$$
\begin{aligned}
\dot{V}_{1 b}(t) \leq & -\frac{\alpha(t)}{2 L^{3 / 2}(t)\left|e_{1}\right|^{1 / 2}} \tilde{e}^{2} \\
& -\sqrt{2}\left(4 L^{-1}+\delta L^{-2}\right)\left(\ell+e_{r}\right)\left|e_{1}\right|^{1 / 2}
\end{aligned}
$$

Now consider the term $\dot{V}_{2}$ from (26). By definition

$$
\begin{aligned}
\dot{V}_{2}(t) & =\frac{d}{d t}\left(\frac{1}{\sqrt{2} \gamma} e_{r}\left(4 L^{-1}+\delta L^{-2}\right) e_{r}\right) \\
& =\frac{1}{\sqrt{2} \gamma}\left(2 e_{r}\left(4 L^{-1}+\delta L^{-2}\right) \dot{e}_{r}-e_{r}\left(4 L^{-2}+2 \delta L^{-3}\right) \dot{L} e_{r}\right)
\end{aligned}
$$

Since $\dot{e}_{r}=\dot{r}$, using $\dot{r}$ from (19) in (39) gives

$$
\begin{aligned}
\dot{V}_{2}(t)= & \sqrt{2}\left(4 L^{-1}+\delta L^{-2}\right) e_{r}\left|e_{1}\right|^{1 / 2} \\
& -\frac{1}{\sqrt{2} \gamma}\left(e_{r}\left(4 L^{-2}+2 \delta L^{-3}\right) \dot{L} e_{r}\right)
\end{aligned}
$$

From (29), (38) and (40), the overall Lyapunov derivative is given by

$$
\begin{aligned}
\dot{V}(t)= & \dot{V}_{1 a}(t)+\dot{V}_{1 b}(t)+\dot{V}_{2}(t) \\
\leq & \frac{1}{2} \zeta^{\mathrm{T}} \psi(t) \dot{L} \zeta-\frac{\alpha}{2 L^{3 / 2}\left|e_{1}\right|^{1 / 2}} \tilde{e}^{2} \\
& -\sqrt{2}\left(4 L^{-1}+\delta L^{-2}\right)\left(\ell+e_{r}\right)\left|e_{1}\right|^{1 / 2} \\
& +\sqrt{2}\left(4 L^{-1}+\delta L^{-2}\right) e_{r}\left|e_{1}\right|^{1 / 2} \\
& -\frac{1}{\sqrt{2} \gamma}\left(4 L^{-2}+2 \delta L^{-3}\right) \dot{L} e_{r}^{2}
\end{aligned}
$$

Note that from (34), $\frac{1}{2} \zeta \psi(t) \dot{L} \zeta \leq 0$ for $\dot{L} \geq 0$ and $L>0$. Therefore

$$
\begin{aligned}
\dot{V}(t) \leq & -\frac{\alpha}{2 L^{3 / 2}\left|e_{1}\right|^{1 / 2}} \tilde{e}^{2}-\sqrt{2}\left(4 L^{-1}+\delta L^{-2}\right) \ell\left|e_{1}\right|^{1 / 2} \\
& -\frac{1}{\sqrt{2} \gamma}\left(4 L^{-2}+2 \delta L^{-3}\right) \dot{L} e_{r}^{2}
\end{aligned}
$$


The function $\dot{V}(\cdot)$ is differentiable other than where $e_{1}=0$. Except within $V_{\phi}=\left\{\left(e_{1}, e_{2}, e_{r}\right) \mid e_{1}=0, e_{2}=0, e_{r}>0\right\}$ motion cannot be sustained on $e_{1}=0$. Consequently $\dot{V}(\cdot)$ is differentiable almost everywhere and the error states converged to a point within the set $V_{\phi}$, and the error system converges to a point where $e_{1}=0$ and $e_{2}=0$.

Proof of Proposition 2: Using the same Lyapunov function candidate in (22), the derivative components as in (26), (27), (29) and (38) are still valid using the adaptive rule (20). Now however $\dot{V}_{2}$ from (39) becomes

$$
\begin{aligned}
\dot{V}_{2}(t)= & \frac{\sqrt{2}}{\gamma}\left(4 L^{-1}+\delta L^{-2}\right) e_{r} \gamma D\left(\left|e_{1}\right|^{1 / 2}\right) \\
& -\frac{1}{\sqrt{2} \gamma}\left(e_{r}\left(4 L^{-2}+2 \delta L^{-3}\right) \dot{L} e_{r}\right)
\end{aligned}
$$

From (29), (38) and (42), the Lyapunov derivative is

$$
\begin{aligned}
\dot{V}(t)= & \dot{V}_{1 a}(t)+\dot{V}_{1 b}(t)+\dot{V}_{2}(t) \\
\leq & \frac{1}{2} \zeta^{\mathrm{T}} \psi(t) \dot{L} \zeta-\frac{\alpha}{2 L^{3 / 2}\left|e_{1}\right|^{1 / 2}} \tilde{e}^{2} \\
& -\sqrt{2}\left(4 L^{-1}+\delta L^{-2}\right)\left(\ell+e_{r}\right)\left|e_{1}\right|^{1 / 2} \\
& +\sqrt{2}\left(4 L^{-1}+\delta L^{-2}\right) e_{r} D\left(\left|e_{1}\right|^{1 / 2}\right) \\
& -\frac{1}{\sqrt{2} \gamma}\left(4 L^{-2}+2 \delta L^{-3}\right) \dot{L} e_{r}^{2}
\end{aligned}
$$

Now there are two cases to consider: If $\left|e_{1}\right|^{1 / 2}>\varepsilon$, then $D\left(\left|e_{1}\right|^{1 / 2}\right)=\left|e_{1}\right|^{1 / 2}$ and so substituting in (43) gives

$$
\begin{aligned}
\dot{V}(t) \leq & -\frac{\alpha}{2 L^{3 / 2}\left|e_{1}\right|^{1 / 2}} \tilde{e}^{2}-\sqrt{2}\left(4 L^{-1}+\delta L^{-2}\right) \ell\left|e_{1}\right|^{1 / 2} \\
& -\frac{1}{\sqrt{2} \gamma}\left(4 L^{-2}+2 \delta L^{-3}\right) \dot{L} e_{r}^{2}+\frac{1}{2} \zeta^{\mathrm{T}} \psi(t) \dot{L} \zeta \\
\leq & 0
\end{aligned}
$$

since $\psi<0$ and $\dot{L} \geq 0$. Notice that (44) is identical to (41). However, if $\left|e_{1}\right|^{1 / 2}<\varepsilon$, then $D\left(\left|e_{1}\right|^{1 / 2}\right)=0$ and substituting in (43) gives

$$
\begin{aligned}
\dot{V}(t) \leq & -\frac{\alpha}{2 L^{3 / 2}\left|e_{1}\right|^{1 / 2}} \tilde{e}^{2}-\sqrt{2}\left(4 L^{-1}+\delta L^{-2}\right)\left|e_{1}\right|^{1 / 2}\left(\ell+e_{r}\right) \\
& -\frac{1}{\sqrt{2} \gamma}\left(4 L^{-2}+2 \delta L^{-3}\right) \dot{L} e_{r}^{2}+\frac{1}{2} \zeta^{\mathrm{T}} \psi(t) \dot{L} \zeta
\end{aligned}
$$

If $r(t)>\delta$ then from (23), $e_{r}(t)>0$ and therefore $\ell+e_{r}>0$ and $\dot{V} \leq 0$ in (45). However if $r(t)<\delta$ then the term $\ell+e_{r}(t)$ in (45) may be negative and therefore

$$
\begin{aligned}
\dot{V}(t) \leq & -\frac{\alpha}{2 L^{3 / 2}\left|e_{1}\right|^{1 / 2}} \tilde{e}^{2}+\sqrt{2}\left(4 L^{-1}+\delta L^{-2}\right) \varepsilon\left(\delta-r_{0}+\ell\right) \\
& -\frac{1}{\sqrt{2} \gamma}\left(4 L^{-2}+2 \delta L^{-3}\right) \dot{L} e_{r}^{2}
\end{aligned}
$$

where $\varepsilon$ is defined in (21) and $r_{0}=r(0)$ which is assumed to satisfy $r_{0}<\delta$, and therefore $\left(\delta-r_{0}+\ell\right)>0$. It is easy to verify if

$$
\tilde{e}^{2}>2 \varepsilon^{2}\left(4+\frac{\delta}{L_{0}}\right)\left(\delta-r_{0}+\ell\right)
$$

where $L_{0}=r_{0}+\ell$ (and consequently $L(t) \geq L_{0}$ for all $t$ ) that $\dot{V} \leq 0$ in (46). Define $\varepsilon_{0}=\sqrt{2 \varepsilon^{2}\left(4+\frac{\delta}{L_{0}}\right)\left(\delta-r_{0}+\ell\right)}$. From the preceding argument, outside the hyper-rectangle

$$
\mathscr{R}=\left\{\left.\left(e_{1}, \tilde{e}, r\right)|| e_{1}\right|^{1 / 2}<\varepsilon,|\tilde{e}|<\varepsilon_{0}, 0 \leq r \leq \delta\right\}
$$

the derivative $\dot{V} \leq 0$ and consequently the states $\left(e_{1}, \tilde{e}, r\right)$ remain bounded. The bound this induces on the evolution of $e_{1}$ depends on the choice of $\varepsilon, \gamma$ and $\ell$, which should be chosen to ensure $e_{1}$ remains small.

\section{REFERENCES}

[1] E. Alcorta-Garcia, A. Zolghadri, and P. Goupil. A novel non-linear observer-based approach to oscillatory failure detection. In European Control Conference, pages 1901-1906, 2009.

[2] E. Alcorta-Garcia, A. Zolghadri, P. Goupil, L. Lavigne, and P. Simon. Nonlinear observer-based OFC detection for A380 aircraft. In Proceedings of the IFAC Symposium SAFEPROCESS '09, Barcelona, pages 47-52, 2009.

[3] H.M. Besch, H. G. Giesseler, and J. Schuller. Impact of electronic flight control system (EFCS) failure cases on structural design loads. Agard report 815, loads and requirements for military aircraft, 1996.

[4] A. Dávila, J. A. Moreno, and L. Fridman. Variable gains super-twisting algorithm: A lyapunov based design. In IEEE Conference on Desicion and Control, pages 968-973, 2010.

[5] P. Goupil. AIRBUS benchmark description. Technical Report ADDSAFE D1.1.3, AIRBUS OPERATIONS SAS, 2010.

[6] P. Goupil. Oscillatory failure case detection in the A380 electrical flight control system by analytical redundancy. Control Engineering Practice, 18(9):1110-1119, 2010.

[7] L. Lavigne, A. Zolghadri, P. Goupil, and P. Simon. Oscillatory failure case detection for new generation AIRBUS aircraft: a model-based challenge. In Proceedings of the Conference on Decision and Control, CDC '08, Cancun, Mexico, pages 1249-1254, 2008.

[8] L. Lavigne, A. Zolghadri, P. Goupil, and P. Simon. Robust and early detection of oscillatory failure case for new generation AIRBUS aircraft. In AIAA Guidance, Navigation and Control Conference and Exhibit, number AIAA 2008-7139, 2008.

[9] A. Levant. Robust exact differentiation via sliding mode technique. Automatica, 34(3):379-84, 1998.

[10] J. A. Moreno and M. Osorio. A Lyapunov approach to second-order sliding mode controllers and observers. In 47th IEEE Conference on Decision and Control, pages 2856-2861, 2008.

[11] R. Pons, C. Jauberthie, L. Trav-Massuys, and P. Goupil. Control surfaces oscillatory failures identification using interval analysis. In The 19th International Workshop on Principles of Diagnosis (DX-08), 2008.

[12] H. Sachs. Fault Investigation and Robust Failure Detection of Oscillating Aircraft Actuation Systems Using Analytical Redundancy. Shaker Verlag GmbH, 2010.

[13] H. Sachs, M. H. Gojny, and U. B. Carl. Robust detection of oscillatory and transient aircraft actuation system failures using analytical redundancy. In SAE 2009 AeroTech Congress \& Exhibition, Seattle, WA, 2009. 